\title{
Course on the simulation of optical components and systems
}

\section{Donald O'Shea}

Donald C. O'Shea, "Course on the simulation of optical components and systems," Proc. SPIE 2525, 1995 International Conference on Education in Optics, (13 October 1995); doi: 10.1117/12.224036

SPIE Event: SPIE's 1995 International Symposium on Optical Science, SPIE. Engineering, and Instrumentation, 1995, San Diego, CA, United States 
Invited Paper

A course on the simulation of optical components and systems

\author{
Donald C. O'Shea \\ Georgia Institute of Technology, School of Physics and \\ Center for Optical Science and Engineering, Atlanta, GA 30332-0430
}

\begin{abstract}
At Georgia Tech the course on Optical Fabrication was dropped from the catalog because the equipment was obsolete in comparison with current technology. In addition, maintenance was becoming difficult and costly. However, there was still a need to provide students with a feel for the design decisions that must be made when specifying a component. We have designed a course to provide students experience in the use of design and simulation programs for optical systems and components. Three types of simulations were explored by the students: lens design, thin film design, and physical optics simulations. This combined lecture and laboratory introduced the student to commercial design packages and provided background on the theory used in the simulations. A number of problems and projects have been devised to provide practice in the use of these programs. This paper is a report on the first offering and evaluations of the course by the students.
\end{abstract}

Keywords: optics education, simulation, optical design, thin film design, physical optics simulation.

\title{
1. INTRODUCTION
}

The Applied Optics curriculum at the undergraduate level at Georgia Tech includes lecture courses on Geometrical and Physical Optics, Laser Physics, and Photographic Physics. The laboratory courses include an Optical Instruments Lab, Advanced Optics Lab, and a new Electro-Optics Lab. The Optical Design and Optical Fabrication courses that are taught in alternate years are combined lecture and lab courses. Beyond the listed courses, students in the Applied Optics track take a number of Special Problems that provide project type experience in the optics laboratory.

The Optical Fabrication course consisted of a series of lectures on thin film design and a laboratory split equally between grinding and polishing of lenses and thin film deposition. With the increasing sophistication of depositing thin films and fabricating optical surfaces, it is becoming difficult to teach the current practice in the Optical Fabrication. Because the equipment in the lab was 20 to 30 years old and had high maintenance costs, the Optical Fabrication course has been removed from the catalog. There is still a need for students to gain experience in the use of state-of-the-art optical design programs. What was needed was a full-fledged course that trains students to use current computer simulations of lenses and thin films.

For many years simulations have been a standard tool of optical designers. In the early days of optical design, the women with accounting ledgers and log-trig tables, who calculated a series of rays through an optical system, were called "computers". More recently the computer has been used increasingly to educate by simulating situations that are difficult to set up in a laboratory or lecture hall. Thus, both teaching and design benefit from the ability to simulate physical systems. Now that tools for optical design have matured and are available on desktop computers and workstations, a course in Optical Simulations becomes a logical extension of the curriculum.

Traditionally, experience with these applications is gained by using the programs in the practice of generating an actual design or by attending a course offered by the software vendor. These courses are intended to teach the new purchaser how to use the program, butthey do not provide much insight into optical design. There are some short courses offered by professional societies that try to remedy this shortcoming, but the societies do not permit practice with the necessary software since this might be considered an endorsement. Considering these modest efforts by professional organizations and the few programs within the educational institutions of the United States that specialize in optics education, there is a need to train students in the theory and practice of these programs. 


\section{DESCRIPTION OF COURSE}

The objective of this new course was to provide experience through a series of lectures and laboratories in numerically modeling optical systems. The systems to be studied included lenses, multilayer films, and propagating beams. The lectures were designed to introduce a number of concepts needed to complete the homework exercises. Because the course material was based on a number of sources, there was no formal text. A set of handouts and notes was provided.

The twice weekly exercises were the heart of the course. An exercise was given each class day and the work was due the next class day. A list of the topics and exercises is given in Table 1 . After evaluating a simple lens, the students were given a series of increasingly more complicated systems including the Hubble telescope, the eye, and diffractive optics. The latter was done because of there is work done in this field at Tech. Because the output from design programs can be voluminous, I asked for specific printouts in each assignment. The students were asked to list their printouts and provide any explanation of results on the first page of their homework set. Later, I asked the students to comment directly on their printouts.

The course is organized around three steps in the design process: specification, evaluation, and optimization. The first of these steps, specification, requires the most physical insight in that it determines the starting point for the design. The control of basic properties of an optical system (focal length, field of view, etc.), and light throughput derives from paraxial analysis of the system. Once these have been determined, the design must be evaluated to see if it meets the required specifications. Finally, the design is modified to optimize its performance. Most of the lecture material was concentrated on the first of these steps. It was in the laboratory that all of the steps were addressed in a series of exercises.

There was one in-lab test and an in-lab final. The homework was worth $60 \%$ of the final grade; the quiz and the final $20 \%$ each.

\section{Software}

Four lens design packages representing four somewhat different approaches were used in the course. CODE V from Optical Research Associates (Pasadena, CA), is a comprehensive program that has both a graphic interface and a command line interface. Once learned, the latter is most useful to rapidly specify and evaluate lenses. OSLO Pro from Sinclair Optics (Fairport, NY) also has a graphics interface that invokes the required commands. There are times when the user must examine the command line to see what must be done to move the program forward. Optikwerk from Optikwerks (Rochester, NY) is more an optical engineering program. It is an icon-based program that uses an optical bench as the basis for the simulation. Components such as scanners, gratings, and prism, a range of sources and detectors are incorporated into the design by placing them on the bench. Although the first two programs use a spreadsheet type entry for the lens prescription (radius, thickness, glass, and clear aperture), the fourth design package, ZEMAX-EE from Focus Software (Tucson, AZ), uses this type of input almost exclusively.

The thin film design package was the Essential Macleod (Tucson, AZ), the work of thin film scientist, Professor Angus Macleod of the University of Arizona. The class started with a version for the Macintosh computer, but a newer version, written for Windows with a graphic interface, provided for us after the course had started, worked better. It also has a spreadsheet input, but most designs can be entered by declaring the materials in the differing layers and then using a short string of characters; the design is easily entered. The reflectance or transmittance of the design as a function of wavelength or angle can be plotted along with other relations.

The beam propagation package we used, Opticalc, was written by Paul Dumas of Macquarie University and runs on Macintosh computers. It is completely graphics based and also uses a lens bench model. A monochromatic source illuminates a mask at one end of a bench. Additional masks, screens (surfaces that can evaluate the beam at any point along the bench, but have no effect on the propagation), and 
lenses can be placed on the bench. The masks can be generated by drawing on them using standard graphics tools or patterns can be imported in a standard graphics format. The program uses Fresnel propagation to compute the electric field and phase from plane to plane and then displays the field or irradiance on a screen placed on the bench. It is a very versatile program that lacks the ability to print directly. By using screen captures the student can make a record of the experiments, but it is awkward.

\section{Hardware}

The Optomechanical Design Lab in the School of Physics at Georgia Tech was begun as part of an initiative to use computers to teach tolerancing of optical system and the optomechanical specification of a system. This lab consists of a network of two accelerated Macintosh IIx's, a Macintosh IIsi, a PowerMac 7100/66, two 486 PC's, a Pentium, and two Apple LaserWriter 360 printers.

The machines are located in a locked room with a punch code lock so that no keys need be given out and access can be controlled from month to month. The students could complete their exercises whenever they had time including nights and weekends. Computers and printers were placed on standard height tables. The students used chairs with adjustable height, because it is my feeling no one should be forced to work at a computer in anything less than a comfortable chair.

\section{RESULTS}

Seven students (4 undergraduates and 3 graduate students) were originally enrolled. Also, a postdoctoral student sat in on the lectures, but did not participate in the exercises. Two of the students were non-physics majors: one a graduate student in ME, the other an undergraduate student in EE. With this number of students the demand on the equipment was not severe. Before the drop date, both of the non-physics majors dropped the course. The graduate was doing a thesis and could not contend with the demands of the course, while the undergraduate felt he did not have sufficient background to complete the exercises.

When the course was originally organized, it was assumed that there would be 10 full weeks of lectures and that 19 exercises could be done in that time. Spring quarter at Tech was not very typical. Besides the loss of one class day because of Dr. Martin Luther King's birthday, there were two other unanticipated days lost, one because of the inauguration of a President of the Institute, and one because of an impromptu celebration that shut down Atlanta for several days. Accordingly some of the material had to be left out. Because of lack of time the exercises in beam propagation were not completed. Instead, only the exercise that introduced the application (Day 16 in Table 1) was given. The work on thin films was completed using new Windows version of the program.

Throughout the course there were hardware and software problems. Each class began with a discussion of these problems. At times this got lengthy. I spent the majority of my time in the course chasing down software discrepancies and printer conflicts. One simply had to chalk this up to this initial offering. Once the impediments were discussed and dealt with, each of the students discussed his experience with the exercise just finished. I lectured on the concepts needed to for the next exercise and answered any questions about the exercise. When it was useful the class continued in the design lab with the demonstration of a program.

\section{Responses}

During the course the students were asked to comment on the each of the exercises. Initially their comments were directed toward the various lens design programs. The initial evaluation of the students was interesting. OSLO Pro was the first or second choice of all of them. But the other first choice, CODE V, was at the bottom of the lists for a number of other students. In the middle were Optikwerks and ZEMAX. As the course progressed, students changed their opinion. CODE $\mathrm{V}$ was considered to be the most powerful, but not the one with which they would choose to begin. OSLO Pro and ZEMAX were favorites. We had memory problems with Optikwerks and eventually had to drop it from the list of packages. All of the students appreciated its interface, but the frustrations of getting an exercise done were too great. 
In the second and third exercises, the differences in the programs were evident to the students. The variation in the scaling and labeling of ray intercept plots and in entering lens prescriptions showed that there was little that could be transferred from program to program. Although I did not ask the question, a number of students remarked that it was a good idea to require them to work on all of the programs rather than let them choose a favorite. Despite some repetition, there is sufficient difference between programs that the practice is warranted. All were impressed with the thin film simulation, particularly after the Windows version was installed. Although their exposure to Opticalc was limited to one session, the students enjoyed using the program. As one student put it: "This is the neatest and easiest program to use."

\section{Final evaluation}

Along with the final exam, the students were asked for an overall evaluation of the course. I asked that the students seal their evaluation when turning it and told them that I would not read it until the course grades had been assigned. There were six questions. I have tried to briefly summarize the responses and add some quotations to give the flavor of the student's reactions. I have not attempted to answer any of the criticisms, since it is their perception of the course that is presented here.

Organization of the course. The students recognized that this was the first time the course was taught and they took that into account. The overall consensus was that the course was "well organized." Holding the classes on Monday and Thursdays made it difficult for some because of weekend obligations, but there is no simple answer for scheduling the lab sessions.

Course content. Most appreciated the range of material. One graduate student wanted Gaussian beam propagation including the modeling of resonators.

Lectures. The students thought the lectures were effective in providing the necessary knowledge to do the exercises. Everyone liked the idea that each person was asked about that day's assignment including any difficulties they encountered or any useful approaches they had found. Most felt the feedback was helpful. As one student noted there was a little too much time spent on the problems with the computers, but that was reduced when a comment and suggestion file was established on one of the machines. There was some criticism that the rationale for various thin film structures was not explained.

Homework exercises. Although one student thought the workload too great in comparison to the credit awarded, others indicated it was a strength of the course. They felt the exercises were instructive and essential. At least one said they were of "Appropriate difficulty and length." Another noted that there was too much at the beginning and that it got better as the quarter progressed. One student suggested that I use of an oral examination in place of a take home exam. Everyone thought the homework was "fairly graded", but most wanted more feedback. Some noted that there were errors or ambiguities in the problem statements that caused some confusion.

Grading. The consensus was that the grading was "fair." Most wanted more comments and a better indication of how close to the ideal result they had gotten.

Facilities. There were some bottlenecks in printing when others were in the lab. One DOS-savvy student pointed out the need for explicit directories for students' files, since some kept leaving their work in his directories. Another student said that he was amazed at how much was provided. He had been at Tech for four years and never been given the use of any computer besides public ones although this was not the first design class he had taken. He also appreciated that extra program keys were obtained for programs and an additional printer was purchased. Most commented favorably on the availability of the lab and on being able to work on assignments at any time. 
Overall evaluation. Everyone said they learned a great deal from the course. Some comments:

"Again I would like to emphasize that I learned a great deal in this course. The large amounts of time I was willing to spend on these assignments is due in no small part to the fact that I found it very interesting."

"Got more out this course than I expected. I'm glad I signed up for the course."

"I knew nothing about lens design coming into this class and now find myself explaining to my friends how things work (the eye, the Hubbell [sic]). To me this was a very valuable class and I am glad I took it."

"I give this course an ' $A$ ' because it has achieved three goals: it has given me a better understanding of optics, a better appreciation of the use of computers in solving problems, and it was fun."

"The class was fun. Would take a special topics course if I was allowed to continue exploring this area."

\section{CONCLUSIONS}

A course on simulation of optical systems has been organized and given. Considering student evaluations and my experience, it fulfilled the objective: to provide experience in simulating optical system through a series of lectures and laboratories. But the course was anything but smooth. The difficulty of maintaining DOS-based systems is an order of magnitude more difficult than it is for Macintosh systems. This reduced the efficiency of the students and put an additional burden on the instructor.

The exercises require additional work to remove inconsistencies and to clarify instructions. Toward the end, I began to break down the instructions into a series of stages that helped the students to organize their efforts. I would probably drop one of the lens design exercises and add an additional beam propagation exercise.

Any similar course must be designed based on the hardware and software available. Despite any extended experience that an instructor may have with a particular piece of software, he or she should do the entire exercise. Even then, the students will find a way to reinterpret your words and the exercise will have to be revised further. Above all, pick examples that engage the students.

\section{ACKNOWLEDGMENTS}

I wish to thank the students who participated in the class. I want to acknowledge an AT\&T Foundation grant that supported the establishment of the Optomechanical Design Lab and the upgrade for this course. I appreciate the long-standing support of the Applied Optics Program in the School of Physics by Hughes Aircraft Company.

I thank the principals and staff of Focus Software, Optical Research Associates, Optikwerks, Inc., Sinclair Optics, The Thin Film Center, and Paul Dumas of Macquarie University for their assistance with their programs, particularly when a rapid response was needed. 
Table 1. Course Outline for Simulation of Optical Systems

Title of Exercise and Day of Lecture Introduction to Optical Design (Day 1)

\author{
Aberration Curves (Day 2.) \\ Definitions of Design Concepts (Day 3)
}

Optimization of a lens design (Day 4)

Chromatic aberration (Day 5)

Mirror systems (Day 6)

Modifying a standard design (Day 7)

Working with the visual system (Day 8)

Diffractive Optics (Day 9)

Test on Lens Design (Day 10)
Topics and Assignments

Overview of Course

History of Design

Software Packages

Homework and Lab: Specifications of a Double Gauss lens

Transverse curves

Field curves

Homework and Lab: Evaluation of the Double Gauss lens

Specifying Systems

System Prescription

First order calculations

Homework and Lab: Enter a biconvex lens; paraxial data

Merit functions: Default vs. Custom

Local vs. Global Optimization: The Monochromatic Quartet

Homework and Lab: Optimize biconvex lens on all programs

Glass choices

The Glass Map

Chromatic aberration curves

Homework and Lab: Split biconvex lens and achromatize.

Afocal Systems

Conic surfaces and spheres

The Hubble Telescope

Homework and Lab: A mirror system of two aspherical surfaces

Lens catalogs

Patent lenses

Classical solutions

Homework and Lab: Take a classical solution and modify it.

The eye as an optical system

The Gullstrand model

Designing an eyepiece

Homework and Lab: An eyepiece evaluation and revision Introduction to Diffractive Optics

The Sweatt Model

Phase functions

Correcting a singlet using a diffractive surface

Homework and Lab: Diffactive correction of a singlet 
Table 1. Course Outline for Simulation of Optical Systems (continued)

Introduction to Thin Film Design (Day 11)

Multilayer AR coatings (Day 12)

Periodic Multilayer Stacks (Day 13)

Narrowband Filters (Day 14)

Tilted elements (Day 15)

Introduction to Opticalc (Day 16)

Spatial Filtering (Day 17)

Gaussian beam simulation or diffractive optics (Day 18)

Final Exam
Fresnel Equations

Matrix Formulation of propagation at an interface

The Quarter-wave Rule

Anti-reflection coatings

Homework and Lab: Single layer AR coatings; different $\lambda s$

Vector picture

Admittance diagram

V-, W-, and more elaborate coats

Homework and lab: Investigate a number of AR coats

HL layers

Short-pass and long-pass filters

$g$-space

Multilayer bandwidths

Homework and lab: Design of multilayer mirrors and pass filters

Fabry-Perot interferometer

$1-, 2-$, and 3- cavity filters

Homework and lab: Generate specific filters

Polarization in multilayer films

Transmission and reflection of an AR coat at $45^{\circ}$

Homework and Lab: Polarizing beamsplitter

Interference and Diffraction patterns

Near-field and far-field patterns

Scalar approximation and validity of calculation

Homework and Lab: Diffraction and interference patterns

Imaging with $4 \mathrm{f}$ systems (1-lens and 2-lens)

Spatial filtering, feature extraction

Homework and Lab: Spatial filtering of simple patterns

Focusing and collimating a Gaussian beam

Effects of aperturing a Gaussian beam

Focusing and collimating a Gaussian beam

No homework

Take home (10 hours permitted) 